\title{
Optical Chiral Negative-Index Metamaterial Design
}

\author{
Do-Hoon Kwon*1, Douglas H. Werner ${ }^{1}$, Alexander V. Kildishev ${ }^{2}$, \\ Vladimir P. Drachev ${ }^{2}$, and Vladimir M. Shalaev ${ }^{2}$ \\ ${ }^{1}$ Department of Electrical Engineering \\ The Pennsylvania State University, University Park, PA 16802, USA \\ ${ }^{2}$ School of Electrical and Computer Engineering \\ Purdue University, West Lafayette, IN 47907, USA
}

\section{Introduction}

A chiral structure refers to a geometrical configuration that cannot be brought into congruence with its own mirror image. Isotropic chiral media are a subset of the general bi-isotropic (BI) media, for which the constitutive relations among electromagnetic vector quantities $\mathbf{E}, \mathbf{H}, \mathbf{D}$, and $\mathbf{B}$ are described by [1]

$$
\begin{aligned}
& \mathbf{D}=\epsilon \mathbf{E}+(\chi+i \kappa) \sqrt{\mu_{0} \epsilon_{0}} \mathbf{H} \\
& \mathbf{B}=(\chi-i \kappa) \sqrt{\mu_{0} \epsilon_{0}} \mathbf{E}+\mu \mathbf{H}
\end{aligned}
$$

where $\chi$ and $\kappa$ are the Tellegen and the chirality parameters, respectively. Recently, a design approach for negative refractive index metamaterials using chiral inclusions [2] has been proposed, and a theoretical chiral negative-index material (NIM) design at optical wavelengths [3] was presented.

A typical NIM design in the optical regime consists of a metal-dielectric-metal sandwich structure with perforations (e.g., see [4]), which is a non-chiral structure. This paper presents a chiral NIM slab design in the near-infrared (near-IR) spectrum. First, the effective material parameter retrieval procedure for a general BI metamaterial is presented, which is based on an extension of the inversion approach for non-chiral metamaterials reported in [5]. Following this, an optical NIM design incorporating magnetic resonators of chiral construction is introduced and the full-wave analysis results for this structure are presented.

\section{BI Material Parameter Inversion}

For a given BI metamaterial slab, we can define the equivalent index of refraction $n_{ \pm}$ and the impedance $z_{ \pm}$for the right-hand (RCP) and the left-hand circular polarized (LCP) components. Their values correspond to those of a homogeneous BI slab that produces the identical transmission and reflection coefficients as the original slab.

Consider a homogeneous BI slab of thickness $d$ which is illuminated by a normally incident plane wave. This is depicted in Fig. 1, where an incident field of RCP/LCP $(+/-)$ polarization impinges on the BI slab from the homogeneous isotropic medium 0 above it having material parameters $\left(n_{0}, z_{0}\right)$. The BI slab is placed on the homogeneous isotropic half-space (medium 2) with material parameters $\left(n_{2}, z_{2}\right)$. Based on the values of the reflection and transmission coefficients $r_{0 \pm}$ and $t_{2 \pm}$ assessed at the top and the bottom interfaces for the two circular polarizations, it is possible to 
recover the material parameters $\left(n_{ \pm}, z_{ \pm}\right)$of the BI slab. Noting that the reflected wave propagating within the BI medium changes its handedness from that of the incident wave, enforcing the continuity of tangential electric and magnetic fields at the two interfaces yields

$$
\begin{aligned}
e^{\mp i k_{ \pm} d} & =\frac{\left(1-r_{0+}\right) / z_{0} \pm\left(1+r_{0+}\right) / z_{\mp}}{t_{2+}\left(1 / z_{2} \pm 1 / z_{\mp}\right)}, \\
e^{\mp i k_{\mp} d} & =\frac{\left(1-r_{0-}\right) / z_{0} \pm\left(1+r_{0-}\right) / z_{ \pm}}{t_{2-}\left(1 / z_{2} \pm 1 / z_{ \pm}\right)},
\end{aligned}
$$

where $k_{ \pm}$denotes the wavenumber for the RCP/LCP component within the homogenized BI medium. From (3)-(4), one obtains a quadratic equation for $1 / z_{ \pm}$and a transcendental equation for $k_{ \pm}$, which are given by

$$
\begin{array}{r}
{\left[\left(1+r_{0+}\right)\left(1+r_{0-}\right)-t_{2+} t_{2-}\right]\left(\frac{1}{z_{ \pm}}\right)^{2}+\frac{2\left(r_{0 \pm}-r_{0 \mp}\right)}{z_{0}}\left(\frac{1}{z_{ \pm}}\right)} \\
-\frac{\left(1-r_{0+}\right)\left(1-r_{0-}\right)}{z_{0}^{2}}+\frac{t_{2+} t_{2-}}{z_{2}^{2}}=0, \\
\cos k_{ \pm} d=\frac{1}{2}\left[\frac{\left(1-r_{0 \pm}\right) / z_{0}+\left(1+r_{0 \pm}\right) / z_{\mp}}{t_{2 \pm}\left(1 / z_{2}+1 / z_{\mp}\right)}+\frac{\left(1-r_{0 \mp}\right) / z_{0}-\left(1+r_{0 \mp}\right) / z_{\mp}}{t_{2 \mp}\left(1 / z_{2}-1 / z_{\mp}\right)}\right] .
\end{array}
$$

Eqs. (5)-(6) can be solved for $z_{ \pm}$and $n_{ \pm}$, which can then be converted into the equivalent parameters $n, z, \chi$, and $\kappa$. During the solution process of (6), proper care should be exercised to select the correct branch for the real part of the arccosine function, similar to what has been done for the non-chiral inversion procedure [5].

\section{Numerical Design Example}

Fig. 2 illustrates the construction of a doubly-periodic chiral NIM in the near-IR regime. One quadrant of the unit cell is illustrated in Fig. 2(a). It is a union of four magnetic resonators, each of which is composed of an alumina $\left(\mathrm{Al}_{2} \mathrm{O}_{3}\right)$ layer of thickness $t_{s}$ sandwiched between two silver $(\mathrm{Ag})$ layers of thicknesses $t_{t}$ and $t_{b}$. The thickness of the alumina spacer is fixed at $t_{s}$ but its vertical location is shifted in the $+\hat{z}$ direction at every $90^{\circ}$ rotation in the counter-clockwise sense. The values of $t_{t}$ and $t_{b}$ are adjusted such that the resonator thickness $t_{t}+t_{s}+t_{b}$ remains constant and the structure is unchanged when viewed from both the $\pm \hat{z}$ directions. A protective alumina layer of $10 \mathrm{~nm}$ thickness is applied on both sides. The first quadrant is rotated by $90^{\circ}$ until the unit cell for the metamaterial shown in Fig. 2(b) is formed. This built-in rotational symmetry guarantees that the reflected and transmitted waves retain circular polarization states. Finally, the metamaterial is placed on a thick glass substrate, which is treated as a half space in the simulation.

The metamaterial is illuminated by circularly-polarized plane waves propagating in the $+\hat{z}$ direction. This electromagnetic scattering problem is rigorously solved using the finite element-boundary integral technique with periodic boundary conditions. Alumina and glass are treated as lossless dielectric materials with constant relative permittivity values of 2.6244 and 2.25 , respectively. The measured permittivity values reported in [6] are used to represent the silver. 
The effective material parameters for an example design are shown in Fig. 3. The values of the geometrical parameters used in this design are chosen to be $p=840 \mathrm{~nm}$, $v=220 \mathrm{~nm}, t_{s}=60 \mathrm{~nm}$, and $t_{t}+t_{b}=140 \mathrm{~nm}$, which makes the total thickness $d$ of the metamaterial equal to $220 \mathrm{~nm}$. The reflection coefficients $r_{0 \pm}$ are found to be the same, which leads to the same reflectances $R_{ \pm}$in Fig. 3(a) and the identical impedances $z_{ \pm}$via (5) for the two circular polarizations as seen in Fig. 3(b). The difference in the transmission coefficients $t_{2 \pm}$ leads to the different results for $n_{ \pm}$ shown in Fig. 3(c). It is observed that a NIM band exists in the $0.978-1.11 \mu \mathrm{m}$ wavelength range for the RCP component only. In a sharp contrast, $n_{-}$steeply increases for the LCP component while $n_{+}$decreases toward negative values as the wavelength is decreased. Therefore, the metamaterial behaves as a NIM only for the RCP polarization. The value of chirality $\kappa$ is plotted with respect to the wavelength in Fig. 3(d). It is also found that the value of $\chi$ (not shown) is equal to zero.

\section{Conclusion}

A material parameter extraction procedure for general BI metamaterials has been presented. The retrieved material parameters correspond to those of the homogeneous BI material slab of the same thickness that produces the same transmission and reflection properties. A doubly-periodic chiral NIM design in the near-IR spectrum has been presented along with the extracted material parameters.

\section{Acknowledgments}

This work was supported in part by the Penn State Materials Research Institute and the Penn State MRSEC under NSF Grant No. DMR 0213623, and also in part by ARO-MURI award 50342-PH-MUR.

\section{References}

[1] I. V. Lindell, A. H. Sihvola, S. A. Tretyakov, and A. J. Viitanen, Electromagnetic Waves in Chiral and Bi-Isotropic Media. Boston: Artech House, 1994.

[2] J. B. Pendry, "A chiral route to negative refraction," Science, vol. 306, pp. 1353-1355, 2004.

[3] V. Yannopapas, "Negative index of refraction in artificial chiral materials," $J$. Phys.: Condens. Matter, vol. 18, pp. 6883-6890, 2006.

[4] G. Dolling, M. Wegener, C. M. Soukoulis, and S. Linden, "Negative-index metamaterial at $780 \mathrm{~mm}$ wavelength," Opt. Lett., vol. 32, no. 1, pp. 53-55, 2007.

[5] D. R. Smith, S. Schultz, P. Markoš, and C. M. Soukoulis, "Determination of effective permittivity and permeability of metamaterials from reflection and transmission coefficients," Phys. Rev. B, vol. 65, p. 195104, 2002.

[6] P. B. Johnson and R. W. Christy, "Optical constants of the noble metals," Phys. Rev. B, vol. 6, pp. 4370-4379, 1972. 


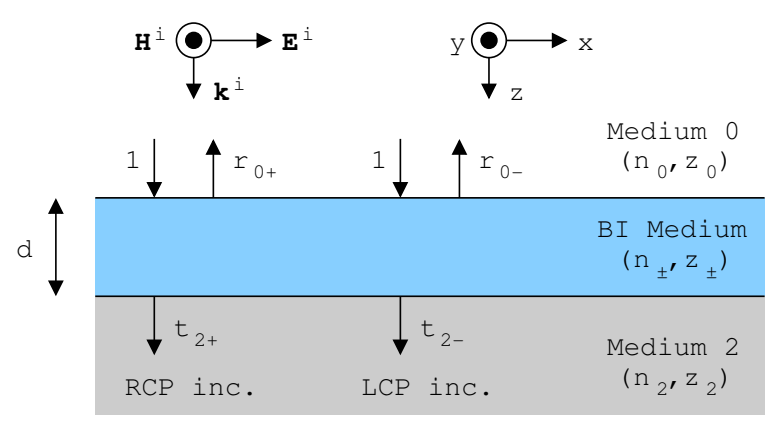

Figure 1: A BI slab under circularly-polarized plane-wave illuminations.

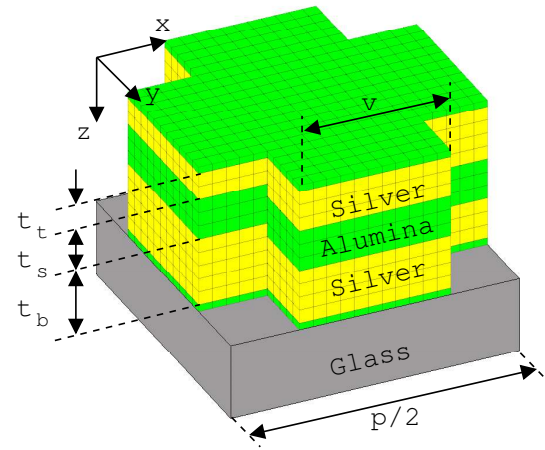

(a)

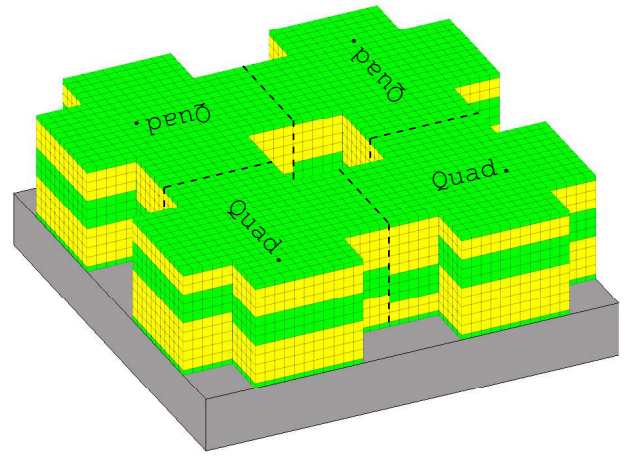

(b)

Figure 2: Construction of the unit cell of a doubly-periodic optical chiral metamaterial: (a) A quadrant, (b) The unit cell.
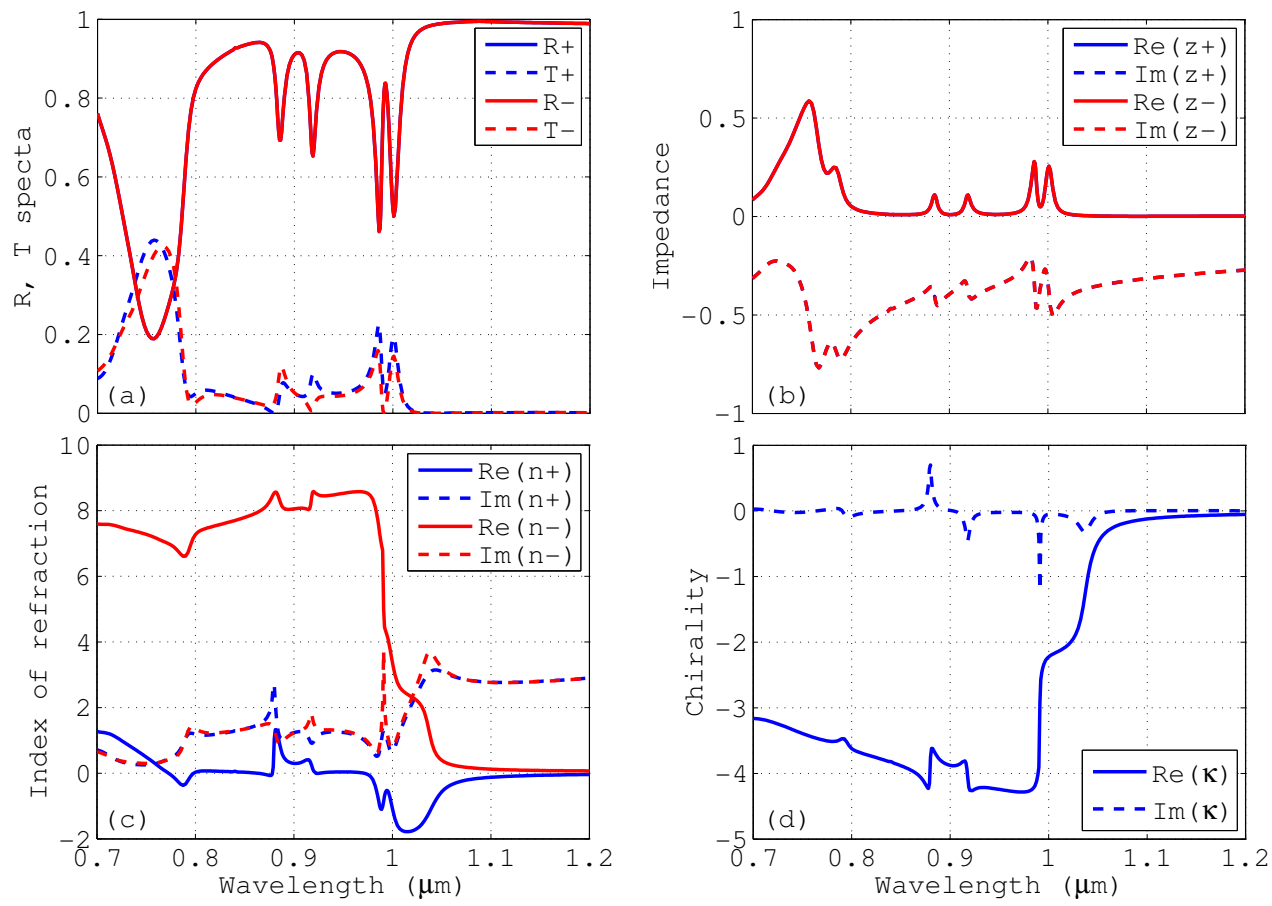

Figure 3: The spectra and material parameters of the chiral optical metamaterial: (a) $R_{ \pm}$and $T_{ \pm}$(reflectance and transmittance), (b) $z_{ \pm}$, (c) $n_{ \pm}$, and (d) $\kappa$. 